\title{
The Status of Early-Type Radial-Velocity Standards
}

\author{
Francis C. Fekel ${ }^{1}$ \\ Center for Automated Space Science and Center of Excellence in \\ Information Systems, Tennessee State University, 330 Tenth Avenue \\ North, Nashville, Tennessee 37203
}

\begin{abstract}
A search for constant velocity B and A stars for use as earlytype radial-velocity standards has continued for 15 years. For the past decade observations of over two dozen stars have been obtained with the KPNO coudé feed telescope, spectrograph, and a CCD detector. Mean velocities are given for $26 \mathrm{~B}$ and A stars plus $\mathrm{HR} 5447$, an F2 V star. Unfortunately, most of the early and mid B-type stars have variable velocities. However, a number of slowly rotating, late B and A-type stars that have constant velocities are identified. From 53 velocities covering nearly 20 years, a preliminary orbit for the early-A star HR $8641=0$ Peg has been determined. It has a period of 10.8 years and a total velocity amplitude of $2.4 \mathrm{~km} \mathrm{~s}^{-1}$.
\end{abstract}

\section{Introduction}

In contrast to the relatively mature situation for late-type radial-velocity standards, as discussed at this conference, that for early-type standards is in its infancy. Morse, Mathieu \& Levine (1991) and Verschueren (1991, 1995) have discussed reduction techniques and the many pitfalls inherent in the measurement of early-type radial velocities. Fekel (1985) presented preliminary results of a search for constant velocity B and A stars. His sample consisted of 29 stars ranging in spectral type from early $B$ to early $A$. Those stars were slow rotators with two-thirds of the sample having $v \sin i \leq 25 \mathrm{~km} \mathrm{~s}^{-1}$. A summary of Fekel's updated results was given by Latham \& Stefanik (1992). The derived mean velocities were from the measurement of individual lines in photographic spectrograms.

This paper examines the status of a revised list of candidate early-type standards, many of which have been observed for at least 15 years. Primarily discussed, however, will be those observations obtained over the past 10 years with a CCD detector. The projected closing within the next two years of the coudé feed telescope at Kitt Peak National Observatory (KPNO) will bring this project to an abrupt conclusion.

\footnotetext{
${ }^{1}$ Visiting Astronomer, Kitt Peak National Observatory. NOAO is operated by AURA, Inc., under cooperative agreement with the National Science Foundation
} 


\section{Observations and Reductions}

From 1989 through 1998 high-dispersion spectroscopic observations were obtained at the KPNO with the coudé feed telescope, coudé spectrograph, and a TI CCD. Some observations, primarily of HR $1389=68$ Tau, HR 2489, and HR $5447=\sigma$ Boo, were made at a wavelength region centered at $6430 \AA$, the typical wavelength region used for late-type stars. Those observations have a wavelength range of over $80 \AA$ and a resolution of $0.21 \AA$. The vast majority of the observations, however, were obtained at a wavelength region centered at $4495 \AA$. Those spectra also have a wavelength range of over $80 \AA$ and a resolution of $0.21 \AA$ with typical signal-to-noise ratios of $150: 1$ to $300: 1$.

Radial velocities were determined for the CCD spectra with the IRAF crosscorrelation program FXCOR (Fitzpatrick 1993). Radial velocities for IAU latetype standard stars were assumed from Scarfe, Batten \& Fletcher (1990). For the red wavelength spectra the wavelength region $6404 \AA$ to $6444 \AA$, which contains five or six moderate-strength lines, was used to determine a cross-correlation velocity. To minimize the spectral-type mismatch, in the blue wavelength region two cross-correlation velocities were obtained. Because the $\mathrm{Mg}$ II line at 4481 $\AA$ is so much stronger than any other metal line in the wavelength region and is the only line measurable in early- and many mid-B stars, its velocity was measured alone. A second cross-correlation velocity was measured for the five Fe II and Ti II lines between 4500 and $4525 \AA$. That group of metal lines can be measured in late-B and A stars. The velocity difference between those two cross-correlation velocities for each star is usually $\leq 0.5 \mathrm{~km} \mathrm{~s}^{-1}$.

A bootstrap procedure has been used to tie the velocities of the early-type stars to the late-type velocity system of Scarfe et al. (1990). The narrow-lined A-stars 68 Tau, HR 2489, and o Peg were initially used as primary early-type velocity standards. However, o Peg was eventually identified as a low-amplitude, long-period binary. The velocities of the primary standards were determined relative to late-type IAU standards, mostly the late-F dwarfs $\iota$ Psc, 10 Tau, and $\beta$ Vir, with observations at both blue and red wavelengths being obtained. Two secondary standards, HR 3383 and HR 8404, whose well-determined mean velocities are tied to the A-star standards, have also been used to detemine cross-correlation velocities when 68 Tau could not be observed.

\section{Results}

Table 1 lists the radial-velocity results of $26 \mathrm{~B}$ and A stars plus HR $5447=\sigma$ Boo (F2 V) for which at least $4 \mathrm{CCD}$ observations have been obtained. Column 1 gives the star's HR number. Columns 2, 3, and 4 list for each star the $V$ magnitude, spectral type, and $v \sin i$, respectively. Column 5 gives the number of CCD observations, column 6 , the mean radial velocity, and column 7 , the standard error of the mean. Column 8 notes those stars whose velocities have been found to be variable.

Not listed in Table 1 are HR 6031, HR 6035, HR 6092, HR 6396, HR 6787, and HR 7891 for which fewer than four CCD observations exist. Those stars have rotational velocities of about $40 \mathrm{~km} \mathrm{~s}^{-1}$ and/or show velocity variability. 
Table 1. Early-type standard-velocity candidates.

\begin{tabular}{rclcrrrl}
\hline HR & $\begin{array}{c}\text { V } \\
(\mathrm{mag})\end{array}$ & $\begin{array}{l}\text { Spectral } \\
\text { Type }\end{array}$ & $\begin{array}{c}v \sin i \\
\left(\mathrm{~km} \mathrm{~s}^{-1}\right)\end{array}$ & $\begin{array}{r}\text { RV } \\
\left(\mathrm{km} \mathrm{s}^{-1}\right)\end{array}$ & $\begin{array}{c}\text { M.E. } \\
\left(\mathrm{km} \mathrm{s}^{-1}\right)\end{array}$ & Note \\
\hline 153 & 3.66 & B2.5 IV & 18 & 9 & 0.34 & 0.18 & \\
675 & 5.03 & A2 V & 18 & 14 & 0.43 & 0.05 & \\
1174 & 5.07 & B3 V & 39 & 6 & 16.21 & 1.28 & var \\
1389 & 4.29 & A2 IV-V & 8 & 28 & 38.97 & 0.12 & \\
1397 & 6.06 & B6 IV & 25 & 5 & 12.04 & 1.00 & var \\
1810 & 4.88 & B2.5 IV & 20 & 7 & 16.50 & 0.27 & \\
2010 & 4.91 & B9.5 V & 21 & 8 & 20.55 & 0.12 & \\
2154 & 5.38 & B5 IV & 20 & 7 & 19.56 & 0.22 & MgII \\
2238 & 4.48 & A1 V & 41 & 7 & -2.01 & 0.95 & var \\
2489 & 6.46 & A9 III & 8 & 14 & 7.68 & 0.07 & \\
2818 & 4.64 & A0 IV & 14 & 10 & 26.81 & 0.15 & \\
3136 & 5.65 & A1 V & 29 & 5 & 44.22 & 0.26 & \\
3383 & 5.81 & A1 V & 4 & 8 & 2.79 & 0.09 & \\
4033 & 3.45 & A1 IV & 44 & 4 & 18.08 & 1.03 & var \\
4187 & 5.80 & A0 V & 14 & 4 & -15.45 & 0.93 & var \\
4295 & 2.37 & A0 IV-V & 40 & 4 & -13.12 & 0.25 & \\
4359 & 3.34 & A2 IV & 18 & 9 & 7.44 & 0.16 & \\
4717 & 5.18 & A3 IV & 44 & 6 & 0.08 & 0.61 & var \\
5447 & 4.46 & F2 V & 8 & 11 & 0.04 & 0.08 & \\
7287 & 5.15 & B8 II-III & 12 & 4 & -2.87 & 1.76 & var \\
7426 & 4.72 & B3 IV & 26 & 4 & -21.20 & 0.86 & var \\
7512 & 6.05 & B8 III & 14 & 5 & -8.84 & 0.11 & \\
7773 & 4.76 & B9.5 V & 18 & 4 & -1.02 & 0.24 & \\
7878 & 6.22 & B8 III & 6 & 4 & -22.65 & 1.24 & var \\
7903 & 6.08 & A0 III & 18 & 5 & -32.62 & 0.37 & \\
8404 & 5.80 & B9.5 V & 4 & 12 & 0.20 & 0.05 & \\
8768 & 6.39 & B2 V & 10 & 7 & -11.37 & 0.80 & var \\
\hline \hline
\end{tabular}




\section{The Orbit of o Peg}

Observations of HR $8641=0$ Peg have been obtained for nearly 20 years. From 1978 through 198715 observations were made at McDonald Observatory and KPNO with photographic plates. Over the past 10 years, 38 CCD spectra were obtained at KPNO. The extensive number of CCD velocities combined with their greater precision resulted in the detection of the long-term variability. When the photographic velocities, given weights of 0.2 relative to the CCD velocities, were added to the solution, the period was refined to 3955 days $=10.83$ years. All the orbital elements are given in Table 2.

\section{Discussion}

Of the five early-B stars in Table 1, only two appear likely to be constant-velocity stars, while the other three are clearly variable. Only two mid-B stars are listed in Table 1. For HR 2154, the star with more observations, the mean radial velocity listed is only from the $\mathrm{Mg}$ II line, which appears to have a constant velocity. Velocities from the weak metal lines on the other hand appear to be variable. Such variability may result from line-profile variations or perhaps from the lower precision of the velocities measured for the weak features. Of the 19 late-B and A stars nearly one-third are clearly variable. Almost one half of the stars appear to be excellent standards, having a standard error of the mean $\leq 0.16 \mathrm{~km} \mathrm{~s}^{-1}$. Four stars with larger errors have only four or five velocities, and so additional observations will be needed to determine their usefulness as standards.

Table 2. Orbital elements of o Peg

\begin{tabular}{cc}
\hline Orbital element & value \\
\hline Period & $3955.0 \pm 237.6$ days \\
Time of periastron & $2446305.8 \pm 429.2 \mathrm{HJD}$ \\
Center-of-mass velocity & $8.48 \pm 0.11 \mathrm{~km} \mathrm{~s}^{-1}$ \\
Velocity semi-amplitude & $1.21 \pm 0.14 \mathrm{~km} \mathrm{~s}^{-1}$ \\
Eccentricity & $0.239 \pm 0.108$ \\
Longitude of periastron & $301.4^{\circ} \pm 31.0^{\circ}$ \\
& \\
$a \sin i$ & $6.36 \pm 0.84 \times 10^{7} \mathrm{~km}$ \\
$\mathrm{f}(\mathrm{m})$ & $0.00066 \pm 0.00024 M_{\odot}$ \\
Standard error of an unit-weight observation & $0.40 \mathrm{~km} \mathrm{~s}^{-1}$ \\
\hline \hline
\end{tabular}

Acknowledgments. I wish to thank Daryl Willmarth and Jeannette Barnes for their extensive help with various aspects of this work over the years. This research has been supported in part by NASA grant NCC5-228 and NSF grants HRD-9550561 and HRD-9706268 to Tennessee State University. 


\section{References}

Fekel, F.C. 1985, in Stellar Radial Velocities, (IAU Coll. No. 88), A.G.D. Philip \& D.W. Latham, Schenectady: L. Davis, 335

Fitzpatrick, M.J. 1993, in Astronomical Data Analysis Software and Systems II (ASP Conf. Ser., 52), R. Hanisch, R. Brissenden, \& J. Barnes, San Francisco: Astron. Soc. Pacific, 472

Latham, D.W., \& Stefanik, R.P. 1992, Trans. IAU, 21B, 269

Morse, J.A., Mathieu, R.D., \& Levine, S.E. 1991, AJ, 101, 1495

Scarfe, C.D., Batten, A.H., \& Fletcher, J.M. 1990, Publ. Dom. Ap. Obs., 18, 21

Verschueren, W. 1991, Ph.D. thesis, Free University of Brussels

Verschueren, W. 1995, Standard Star Newsletter, 18, 6

\section{Discussion}

Scarfe: I have about ten observations of 68 Tauri, measured as nearly as possible on the system of Scarfe, Batten \& Fletcher (1990). Our mean values differ by $80 \mathrm{~m} \mathrm{~s}^{-1}$, less than the uncertainty of either.

Fekel: This is excellent news.

Popper: As I understand it, your velocity system is based entirely on the $\mathrm{Mg}$ II $4481 \AA$ line. In the laboratory this line is a close doublet. Might not the relative intensities of the components depend on temperature and/or density, introducing potential systematic effects into the velocity system?

Fekel: For early B stars only the Mg II line was measured for velocity, since the Fe II and Ti II lines are too weak. The Mg II line is indeed a close doublet, whose components, as I recall, have nearly equal intensity. I have not checked to see how the intensities vary with temperature and/or density.

Mazeh: Don't you lose a lot of spectral information by not using the whole observed region of the spectrum for cross-correlation?

Fekel: I wanted to be consistent from star to star, by using a particular set of spectral lines in a clear wavelength region, to avoid blending at higher rotational velocities. I get similar results from the whole region. 